\title{
Realization of A Regional Terrestrial Reference Frame: A GPS Campaign
}

\author{
Ziqing Wei \\ Xian Research Institute of Surveying and Mapping \\ 1 Yanta Mid Road \\ Xian, Shaanxi 710054, P.R.C.
}

A terrestrial reference frame can be defined by the coordinates of a set of stations on the surface of the earth. It is possible to realize a reference frame through the GPS positioning. In this sense two basic approaches are available to realizing a terrestrial reference frame using GPS: absolute and relative. By the absolute approach we mean the point positioning, whereas by the relative approach is meant the relative positioning. At the present day the absolute approach should realize a reference frame at a meter or submeter level precision. By contrast, the relative approach could realize a reference frame with a centimeter or even better precision.

A GPS survey campaign took place between May 1991 and June 1992 in China, with the objective being, among other things, to establish a regional geocentric reference frame. During the campaign 10 MINIMAC-2816 receivers occupied over 40 well-distributed stations constituting a geodetic network on a national scale. The network was divided into 11 interlocking subnetworks with up to 10 stations each. Normally some dozens of three-hour sessions were observed during each subnetwork occupation. A great volume of data was collected. Data reduction is under way.

As the first step, using a subset of data the point positioning has been carried out utilizing carrier phase measurements. The coordinates were obtained for 35 stations as the mean of individual session solutions, with the standard errors at the submeter level. A comparison was made between GPS and SLR( in ITRF90) coordinates for the available SLR stations SHANGHAI and WUHAN, both near to a GPS station, with the results as follows:

$\begin{array}{lcrr}\text { GPS-SLR } & \text { X(m) } & \text { Y(m) } & \text { Z(m) } \\ \text { SHANGHAI } & 0.244 & -0.795 & -0.539 \\ \text { WUHAN } & -0.277 & 0.310 & -0.636\end{array}$

Since the broadcast ephemeris was held fixed in the computation the reference frame, as defined by the coordinates of these 35 stations, should largely align with the WGS 84 reference frame.

The simultaneous adjustment of the whole network at raw phase observation level is being undertaken. At this writing, a trial adjustment, involving 11 stations and 86 observing sessions, has been completed. It shows that using the relative approach the regional terrestrial reference frame could be realizable with the centimeter level precision, which would be of even greater value to geodesy and geodynamics. 